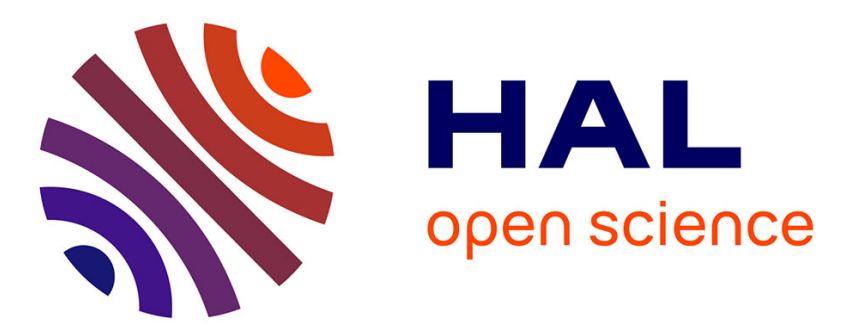

\title{
Un cas de fabrication collective à Saqqâra? La table d'offrandes de Setjou
}

Rémi Legros

\section{To cite this version:}

Rémi Legros. Un cas de fabrication collective à Saqqâra? La table d'offrandes de Setjou. Journal of the American Research Center in Egypt, 2013, 49 (1), pp.153-166. 10.5913/0065-9991-49-1-153 . halshs-01435422

\section{HAL Id: halshs-01435422 \\ https://shs.hal.science/halshs-01435422}

Submitted on 13 May 2020

HAL is a multi-disciplinary open access archive for the deposit and dissemination of scientific research documents, whether they are published or not. The documents may come from teaching and research institutions in France or abroad, or from public or private research centers.
L'archive ouverte pluridisciplinaire HAL, est destinée au dépôt et à la diffusion de documents scientifiques de niveau recherche, publiés ou non, émanant des établissements d'enseignement et de recherche français ou étrangers, des laboratoires publics ou privés. 


\title{
Un cas de fabrication collective à Saqqâra? La table d'offrandes de Setjou
}

\author{
RÉMI LEGROS
}

\begin{abstract}
The offering table under consideration is now in a private collection, but some criteria can assign its origin to Saqqara during the end of the FIP. Some paleographical peculiarities seem to indicate that it was made by at least two different craftsmen. This hypothesis constitute the starting point of a discussion on the localization of workshops and organization of tasks.
\end{abstract}

La table d'offrandes présentée dans cette communication appartient aujourd'hui à un collectionneur privé. ${ }^{1}$ Les circonstances de sa découverte nous sont inconnues. Plusieurs parallèles nous permettent néanmoins de préciser sa date et sa provenance. Certaines particularités originales, principalement sur la paléographie, fournissent aussi l'occasion d'émettre quelques considérations sur les modalités de fabrication des monuments de culte privé. ${ }^{2}$

\section{La table d'offrandes de Sétjou}

La table se présente sous la forme traditionnelle qui devient canonique à partir de la $6^{\mathrm{e}}$ dynastie, ${ }^{3}$ à savoir un pain-hetep, disposé pointe en bas, et deux bassins verticaux, répartis de part et d'autre du pain. Les inscriptions, qui sont le plus souvent portées sur la natte, sont ici cantonnées à la surface du fond, certaines encadrées par des lignes pour former un cadre périphérique. ${ }^{4}$ Le pain lui-même est inscrit et porte une représentation du défunt, assis derrière une table d'offrandes couverte de pains. La taille de l'objet est importante $(98 \mathrm{~cm} \times 47 \mathrm{~cm}),{ }^{5}$ les finitions soignées et la gravure des signes précise, ce qui indique certainement un monument d'une qualité supérieure, ayant appartenu à une personne de rang élevé.

${ }^{1}$ L'objet est connu par la vente Tajan du 30 octobre 2002, tenue à l'Hôtel Drouot, à Paris (catalogue de vente p. 8-9, lot $\mathrm{n}^{\circ} 70$ ). Nous remercions la propriétaire de cette table, qui a souhaité garder l'anonymat, de nous avoir fourni toutes les facilités pour en préparer la publication.

${ }^{2}$ Pour ce deuxième aspect, le titre de l'article fait référence à une autre communication sur ce thème et dont nous souhaiterions, modestement, suivre les traces (cf. P. Der Manuelian, "A Case of Prefabrication at Giza? The False Door of Inti," JARCE 35 [1998], 115-27).

${ }^{3}$ M. Mostafa, Untersuchungen zu Opfertafeln im Alten Reich, HÄB 17 (Hildesheim, 1982), 123-24; R. Hölzl, Ägyptische Opfertafeln und Kultbecken. Eine Form- und Funktionsanalyse für das Alte, Mittlere und Neue Reich, HÄB 45 (Hildesheim, 2002), 15, 23-24 (type $\mathrm{B}+\mathrm{C})$.

${ }^{4}$ Notons dès à présent que ce cadre est avant tout esthétique et n'indique pas un ordre de lecture particulier puisque les colonnes encadrées ne sont pas à lire dans la continuité des lignes.

${ }^{5}$ Pour cette période, la longueur moyenne des tables d'offrandes est un peu inférieure à $40 \mathrm{~cm}$. 

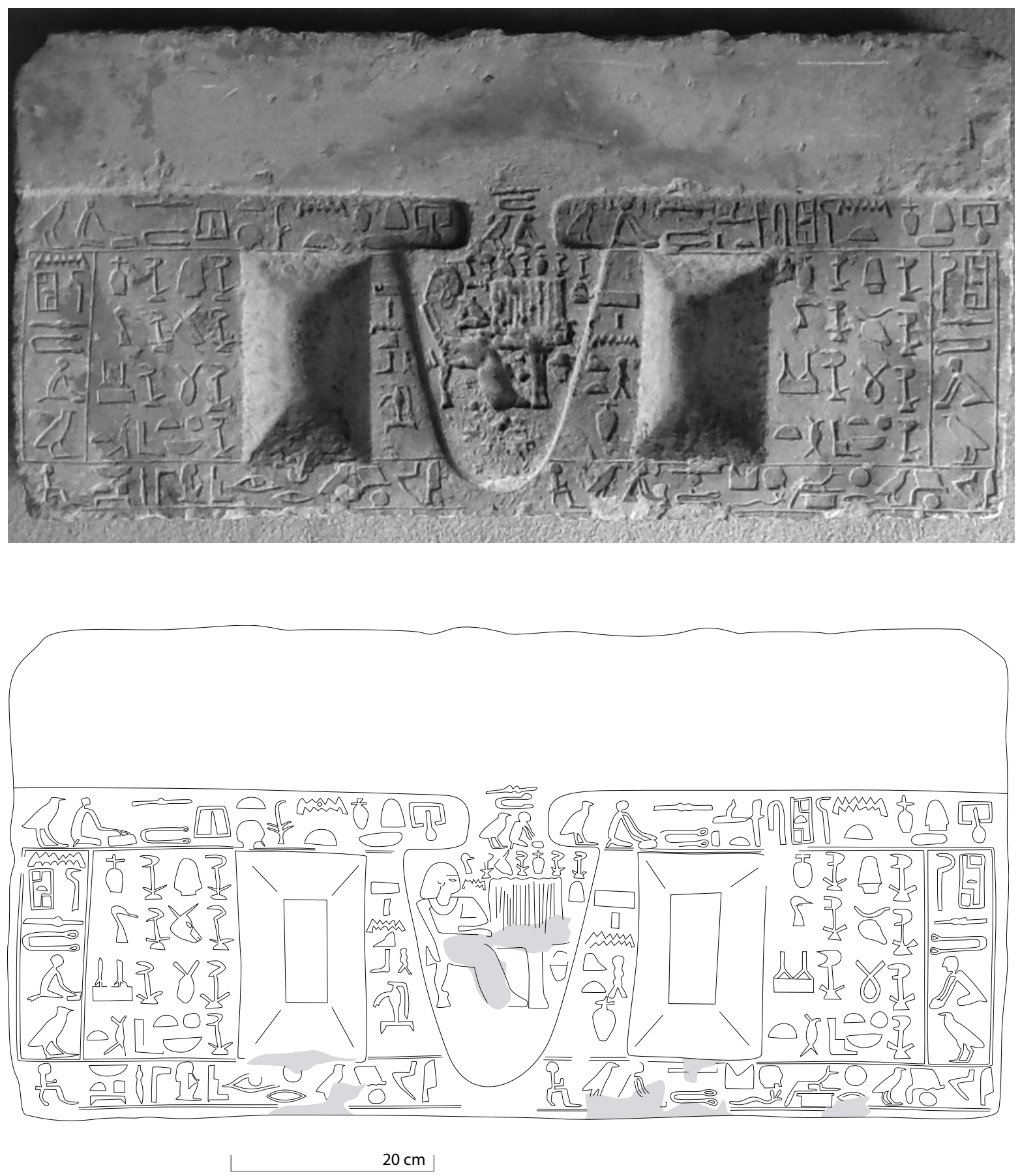

Fig. 1. La table d'offrandes de Sétjou (photographie du propriétaire, dessin de l'auteur d'après photographies de détail). 
L'érosion constatée à l'endroit de la figure est fréquente sur les tables d'offrandes et le plus souvent circonscrite au niveau du pain. Il s'agit d'une marque d'usage, liée au dépôt d'éléments acides qui rongent le calcaire (fig. $1 \mathrm{a}-\mathrm{b}){ }^{6}$

\section{Translittération et traduction}

1. Ligne sous la natte: prt-hrw t-hnqt nt (a) hq3-hwt (b) smr w'ty Sthw - prt-hrw t-hnqt nt (c) hry-tp (d) $n s w t ~ S t \underline{t} w$

2. Pain: $S \underline{t} w$ h 3 t $h 3$ h hnqt $h 3 j h w$ h $33 p d w n$ (e)

3. à côté des bassins: $\check{s} n h n q t-\check{s} n q b h$ (f)

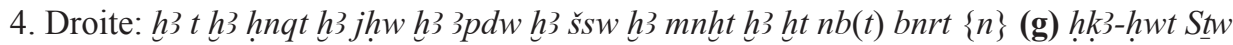

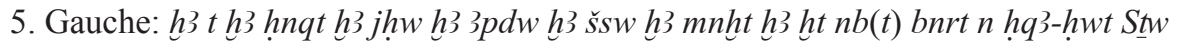

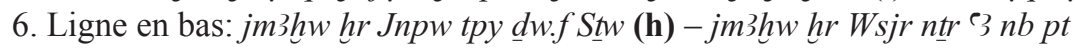

1. Une offrande invocatoire de pain-bière pour le chef de district, l'ami unique Sétjou - une offrande invocatoire de pain-bière pour le sous-ordre du roi, Sétjou.

2. Sétjou. Mille pains, mille bières, mille bœufs, mille volailles.

3. Bassin pour la bière - bassin pour la libation.

4. Mille pains, mille bières, mille bœufs, mille volailles, mille albâtres, mille étoffes, toute chose douce \{pour\} le chef de district, Sétjou.

5. Mille pains, mille bières, mille bœufs, mille volailles, mille albâtres, mille étoffes, toute chose douce pour le chef de district, Sétjou.

6. le pensionné auprès d'Anubis qui est sur sa montagne, Sétjou - le pensionné auprès d'Osiris, le grand dieu maître du ciel.

\section{Commentaire}

a- La formule prt-hrw suivie de $n t$ est maintenant bien connue. ${ }^{7}$ Elle indique assez clairement une origine memphite pour notre monument. ${ }^{8}$ Elle permet également de proposer une datation préliminaire entre la fin du règne de Pépy II et le début du Moyen Empire. ${ }^{9}$ Le doublement de cette formule sur la natte est tout à fait atypique et laisse supposer une conception de ce monument comme deux moitiés distinctes (voir infra).

b- Le signe $h w t(\mathrm{O} 7)$ est tout à fait particulier, avec ses deux carrés intérieurs, l'un en bas, à l'avant, et l'autre dans l'angle opposé. Cette variante, avec le signe $t$ au milieu, ne m'est connue par aucun parallèle, mais se rapproche de certaines formes rares sans le complément phonétique, ${ }^{10}$ dont les attestations datent pour la plupart de la transition entre l'Ancien et le Moyen Empire.

\footnotetext{
${ }^{6}$ Sur l'évolution des usages rituels liés aux tables d'offrandes, voir R. Legros, Stratégies Mémorielles, TMO (Lyon, à paraître).

${ }^{7}$ L. Postel, "Une variante septentrionale de la formule d'offrande invocatoire à la Première Période Intermédiaire: $p r t-h r w$ nt," dans C. Berger et L. Pantalacci, éds., Des Néferkarê aux Montouhotep: travaux archéologiques en cours sur la fin de la VI dynastie et la Première Période Intermédiaire: actes du colloque CNRS-Université Lumière-Lyon 2, tenu le 5-7 juillet 2001, TMO 40 (Lyon, 2005), $255-78$.

${ }^{8}$ En plus des références données par L. Postel (cf. note précédente), la Mission archéologique française de Saqqâra a découvert près d'une centaine d'autres attestations de cette variante sur la nécropole de Pépy ${ }^{\text {er }}$, actuellement en cours d'étude.

${ }^{9}$ R. Legros, "Approche méthodologique pour une datation des tables d'offrandes de la Première Période intermédiaire," BIFAO 108 (2008), 239.

${ }^{10} \mathrm{~J}$. Moreno García, Hwt et le milieu rural égyptien du IIIe millénaire. Économie, administration et organisation territoriale, BEHE 337 (1999), 25, fig. 2 [36]= ramesside et fig. 2 [46]= PPI. On retrouve encore cette variante sur une stèle abydénienne de la $8^{\mathrm{e}}$ dynastie (Louvre C 198+E 3128: C. Ziegler, Catalogue des stèles, peintures et reliefs égyptiens de l'Ancien Empire et de la Première Période Intermédiaire, vers 2686-2040 avant J.-C. [Paris, 1990], 200-203 et E. Brovarski, "Abydos in the Old Kingdom and First Intermediate Period, part I," dans C. Berger, G. Clerc, et N. Grimal, éds., Hommages à Jean Leclant, BdE 106/1 [Cairo, 1994], 100) et sur
} 
c- La graphie du signe $n$, irrégulière et avec les extrémités qui descendent au delà des vagues intérieures, n'est pas rare pour cette période. ${ }^{11} \mathrm{Il}$ est intéressant en revanche de relever la graphie sur la moitié gauche de la natte, qui a laissé un reliquat à l'intérieur du signe. Il témoigne d'une évolution progressive sous la PPI qui tend à systématiser le décalage entre les bords supérieurs et inférieurs. ${ }^{12}$ Il indique encore que ce changement est dû - au moins en partie - à des considérations techniques, avec le passage d'un signe gravé d'un seul tenant vers un signe dont les deux bords sont gravés de manière successive. ${ }^{13}$

d- Le signe du billot T28 présente un détail intérieur original. Au lieu des deux triangles affrontés habituels, il n'y a ici qu'une seule pointe. ${ }^{14}$ Parfois, cette pointe traverse l'ensemble du signe et des attestations sont connues à partir de la fin de la $6^{\text {e }}$ dynastie et au moins jusqu'à la fin de la PPI ${ }^{15}$ Le fait que la pointe, ici, ne traverse pas l'ensemble du signe est beaucoup plus rare: en dehors d'une attestation à Balat, très originale ${ }^{16}$ cette variante est attestée essentiellement à Dendéra sous la $11^{\mathrm{e}}$ dynastie. ${ }^{17}$ Le signe $t p$ est posé sur la ligne d'encadrement de l'inscription. Il ne s'agit pas nécessairement d'une maladresse: sur certains monuments de la PPI les signes sont disposés ainsi de manière beaucoup plus systématique, ce qui pourrait indiquer une volonté délibérée, une mode.

e- Sur le pain, le $n$ qui suit la liste d'offrandes pourrait renvoyer à la figuration du dessin, selon le principe bien étudié par H. Fischer. ${ }^{18} \mathrm{Il}$ s'agit toutefois d'un procédé tout à fait atypique dans ce contexte et je n'en connais aucun autre exemple.

f- La présence d'inscriptions pour expliciter le rôle des bassins est fréquente. En revanche, l'indication près du bassin de gauche, qui fait référence à la libation $(q b h)$, est tout à fait atypique, l'habitude étant de mentionner plutôt l'eau $(\mathrm{mw}) \cdot{ }^{19}$ Quelques attestations ambiguës existent pourtant, qui associent le déterminatif du vase aux trois signes de l'eau. La lecture peut alors varier selon le sens des signes: par-

une autre stèle, à Naga ed-Deir, datée de la fin de la $9^{\text {e }}$ dynastie (Caire, JdE, 45969: E. Brovarski, The Inscribed Material of the First Intermediate Period from Naga-Ed-Dêr, UMI Dissertation [1989], 685). Une variante proche avec un seul carré dans l'angle supérieur arrière se trouve sur une stèle de Naga ed-Deir de la $9^{\mathrm{e}}$ dynastie (Caire, JdE 43755: idem, 722) ainsi que sur la stèle thébaine d'Antef II (J.-J. Clère et J. Vandier, TPPI, 11). Voir également F. Servajean, Le tombeau de Nakhtamon (TT 335) à Deir al-Medina. Paléographie, PalHiéro 5 (2011), 75, §147 avec bibliographie antérieure.

11 Pour ce signe à la fin de l'Ancien Empire, voir notamment P. Collombert, Le tombeau de Mérérouka. Paléographie, PalHiéro 4 (2010), 101, § 182 .

${ }^{12}$ Le décalage entre les deux bords du signe n'indique pas nécessairement un signe mal gravé (même si c'est souvent le cas). Il y a bien une évolution dans la conception même, comme en témoignent des exemples d'une grande qualité d'exécution (voir par exemple, pour la $9^{e}$ dynastie, la stèle de Méni à Dendéra, MFA 98.1034).

${ }^{13}$ Ce que l'on pourrait appeler le ductus dans la pierre mériterait une étude plus complète et systématique. Des découvertes sont encore possibles dans ce domaine et expliqueraient certaines évolutions des signes, notamment durant la PPI.

${ }^{14}$ Sur l'interprétation de ce motif comme un canal d'évacuation, voir P. Collombert, Mérérouka, 139, §236.

${ }^{15}$ On retrouve cette variante notamment sous la $8^{\mathrm{e}}$ dynastie à Abydos (CGC 1615: H. Fischer, "The Cult and Nome of the Goddess Bat," JARCE 1 [1962], 17-18, fig. 3); sous la $9^{\mathrm{e}}$ dynastie à Dendéra (Stèle de Rési: W. Petrie, Dendereh, pl. 10, $3^{\mathrm{e}}$ ligne, à droite), Moallah (Fischer, Dendera, n. 583), Naga ed-Deir (LMA 6-19881: E. Brovarski, Naga-Ed-Dêr, 609-10), et Naqada (CGC 20500: Fischer, Coptite Nome, pl. 18); sous la 10e dynastie à Naga ed-Deir (Hanovre 1971.10: J. Vandier, "Quatre stèles de la fin de l'Ancien Empire et de la première période intermédiaire," $R d E 2$ [1936], pl. 1.2). Cette variante se retrouve un peu avant sur les inscriptions rupestres.

${ }^{16}$ Stèle de Déchérou (J. Osing, M. Moursi, Do. Arnold, O. Neugebauer, R. Parker, D. Pingree et M. Nur-el-Din, Denkmäler der Oase Dachla. Aus dem Nachlass von Ahmed Fakhry, AVDAIK 28 [Mainz, 1982], pl. 57, n 14). Pour une datation sous Pépy I ${ }^{\mathrm{er}}$, voir G. Castel, N. Cherpion et L. Pantalacci, Balat V. Le mastaba de Khentika: Tombeau d'un gouverneur de l'Oasis à la fin de l'Ancien Empire, FIFAO 40 (Cairo, 2001), 4-5.

${ }_{17}$ En particulier MFA 98.1043: R. Leprohon, CAA, Museum of Fine Arts, Boston, Fascicle 2, Stelae I, The Early Dynastic Period to the Late Middle Kingdom (Mainz, 1985), 31-32.

${ }^{18}$ H. Fischer, L'Écriture et l'art de l'Égypte ancienne. Quatre leçons sur la paléographie et l'épigraphie pharaoniques (Paris, 1986), 28.

${ }^{19}$ Lorsque la fonction des bassins est explicitée, c'est celui de droite qui est destiné à l'eau dans trois quarts des attestations (contrairement au cas présent). 
fois, le signe du vase (W15/16) ${ }^{20}$ est placé après l'eau, dans un rôle de déterminatif et l'on aurait alors affaire à une caractérisation de cette eau, pure ou spécifique à la libation. ${ }^{21}$ Mais il existe également des attestations de cette expression où les signes sont inversés et il faut alors considérer qu'il s'agit bien du mot $q b h$, comme c'est le cas sur la table de Sétjou. ${ }^{22}$

g- La mention de toutes offrandes douces est extrêmement rare dans ce contexte. ${ }^{23}$ La rareté de cette formulation explique peut-être l'erreur du graveur et l'échange des signes $b$ et $s t$ sur la moitié droite. ${ }^{24}$ Après la liste d'offrandes, en haut de la colonne de droite, un $n$ était sans doute attendu avant le titre, comme sur celle de gauche. Il s'agit probablement d'un oubli.

h- Le signe A50 joue sans doute le rôle de déterminatif du nom dans la partie droite de l'inscription. ${ }^{25}$ En revanche, pour la partie gauche, il est incongru. Peut-être le nom du défunt a-t-il été omis par manque de place. La représentation d'un bras horizontal est bien attestée à la fin de l'Ancien Empire. ${ }^{26}$ Les formes les plus précoces font sortir le bras au niveau de l'aine. Lorsqu'il semble sortir du tronc, comme ici, c'est parfois la marque d'une datation plus avancée dans la PPI. ${ }^{27}$

\section{Typologie et datation}

L'absence d'inscription sur la natte constitue une caractéristique tardive au sein de la PPI,,$^{28}$ en particulier lorsqu'elle est large comme c'est le cas ici. ${ }^{29}$ Le fait que l'inscription débute par la formule prt$h r w$ et non par htp-dj nswt est une caractéristique presque exclusive à la période 7 de notre matrice. ${ }^{30}$

La multiplication des offrandes semble caractéristique de la PPI pour les tables d'offrandes. À partir de six, ce critère s'avère postérieur à la $6^{\text {e }}$ dynastie. Au delà de ce nombre, il est spécifique à la période héracléopolitaine. ${ }^{31}$

Sur le pain, la figuration du défunt est conventionnelle et bien proportionnée. La position du bras gauche, replié sur le buste, correspond à une attitude fréquente à partir de la PPI sur les tables d'offrandes. ${ }^{32}$ La présence d'un vase dans sa vasque, sous la table à l'avant du pied, permet de réduire

\footnotetext{
${ }^{20}$ Le choix du signe W16 (avec support), sur la table de Sétjou, est rare aux périodes anciennes. Il est néanmoins attesté dès les premières dynasties: Fischer, Dendera, 197. Unique à ma connaissance, le support ressemble ici au signe du ciel. Pour des attestations plus tardives, voir F. Servajean, tombeau de Nakhtamon, 116, §230. Il existe au moins une attestation du signe W15 avec une lecture hs: M. Valloggia, Le monument funéraire d'Ima-Pepy/Ima-Meryrê. Balat 4, FIFAO 38.1 (Cairo, 1998), 77, fig. 15.

${ }^{21}$ Cette variante est connue par trois tables de la nécropole de Pépy I ${ }^{\text {er }}$ Sc-197, et 78 no-0589 (C. Berger-El Naggar et A. Labrousse, "La tombe de Rêhérychefnakht à Saqqâra-Sud, un chaînon manquant?," BSFE 164 [2005], 21, fig. 8) et 78 no-1579. On retrouve cette variante également sur la table CGC 57016, provenant de Saqqâra, et dont le type est tout à fait similaire à celui de la table de Sétjou. Cette table est mentionnée par H. Fischer, justement pour la mention en question, qu'il propose de traduire cool water ("An Invocatory Offering Basin of the Old Kingdom," MDAIK 47 [1991], 127).

${ }^{22}$ Une seule attestation dans le corpus de la MafS, très lacunaire (78 no-0512). On regardera par ailleurs les tables de Kom el-Fakhry, qui portent sur le fond la représentation d'un vase-qbh: K. Daoud, Necropoles Memphiticae. Inscriptions from the Herakleopolitan Period, Studies in Calligraphy and Writings 14 (Alexandria, 2011), 318-20, § 8.4.1 et 8.4.2.

${ }^{23}$ Une seule autre table à ma connaissance porte cette expression: CGC 1335, de date peut-être similaire (notamment en raison du signe de la colline dans la graphie de $\underline{d} s r)$. Voir par ailleurs G. Lapp, Die Opferformel des Alten Reiches unter Berücksichtigung einiger späterer Formen, SDAIK 21 (1986), 134-45; W. Barta, Opferformel, 58.

${ }^{24}$ Sur la moitié gauche, le signe est en lacune, mais la partie verticale semble descendre trop bas pour permettre une lecture

${ }^{25}$ Sur cet usage du signe A50, voir H. Fischer, "Redundant Determinatives in the Old Kingdom," MMJ 8 (1973), 7-25.

${ }^{26}$ Collombert, Mérérouka, 20-21, \$ 23-24.

${ }^{27}$ Cette forme apparaît dès la $6^{\text {e }}$ dynastie, mais se développe ensuite surtout durant la période héracléopolitaine (Brovarski, Naga-Ed-Dêr, 521-22). Lorsqu'un seul bras est visible, il est possible en fait que les deux soient confondus (idem, 652).

${ }^{28}$ Dans le corpus de la nécropole de Pépy I ${ }^{\text {er }}$, plus de $90 \%$ des attestations de ce critère datent du dernier tiers de la PPI.

${ }^{29}$ On considère comme large une natte dont la hauteur est supérieure à un quart de la hauteur totale de la table.

${ }^{30}$ R. Legros, "Approche méthodologique," 238-39.

${ }^{31}$ R. Legros, "Approche méthodologique," 245.

32 Principalement les périodes 3 à 7 de la sériation du BIFAO.
} st. 
cet intervalle à la fin de la période ${ }^{33}$ Le bras droit présente une caractéristique dans le rendu final, avec un décalage entre l'intérieur et l'extérieur du signe: l'avant-bras et la main sont représentés au dessus du volume représenté par le bras et le coude. Il ne s'agit pas à proprement parler d'une erreur dans la mesure où cette caractéristique se retrouve sur d'autres monuments et semble donc volontaire. ${ }^{34}$

La présence de traits qui forment un cadre sur le pourtour du fond trouve des parallèles sur plusieurs tables de la fin de la PPI et annonce peut-être certaines formes à bandeau périphérique du Moyen Empire. En outre, cette particularité pourrait trouver un parallèle dans le développement, durant la même période, d'une colonne encadrée sur le centre du pain. ${ }^{35}$ Sur la base de ce critère il est possible de distinguer un groupe de tables qui semblent former un ensemble cohérent, tant sur la datation - la dernière phase de la PPI - que sur la qualité générale, qui est toujours très bonne. La table d'offrandes la plus proche de notre monument - par le style - est celle publiée au Catalogue Général sous le n 57016, découverte à Saqqâra, dans un secteur attribué à la période héracléopolitaine. ${ }^{36}$

Considérant l'ensemble des remarques énumérées ci-dessus, il est assez probable que cette table d'offrandes date de la dernière phase de la PPI. ${ }^{37}$ À cette époque, les objets de qualité comme celui-ci sont quasiment tous originaires du site de Saqqâra et - si l'on ajoute à cela la formule prt-hrrw nt - c'est très vraisemblablement de là que provient aussi la table de Sétjou.

\section{Le propriétaire du monument}

Le nom Sthw est connu à l'Ancien comme au Moyen Empire. ${ }^{38}$ La graphie est remarquable en raison du déterminatif, qui ne représente pas, comme on pourrait l'attendre, un vase au dessus de la tête, en train de se déverser. ${ }^{39}$

Une forme équivalente de ce signe a déjà été retrouvée par la MafS (fig. 2). Sur ce bloc, retrouvé errant, l'ordre des deux premiers signes avait pu laisser penser qu'il s'agissait d'un autre nom, mais notre table d'offrandes permet maintenant d'assurer qu'il s'agit en fait d'une simple inversion graphique. ${ }^{40}$ Notons dès à présent que ce signe est représenté de deux manières sur la table d'offrandes: parfois avec un seul bras, parfois avec les deux.

33 R. Legros, "Approche méthodologique," 244.

34 Voir par exemple la table 78 no-0106 publiée par C. Berger-El Naggar, dont la date est sans doute assez proche de notre monument, en raison de la présence de vases sous la table et le fauteuil ("Cultes des reines et cultes privés dans le cimetière de la famille royale de Pépy I ${ }^{\mathrm{er}}$," dans C. Berger-El Naggar et L. Pantalacci, eds., Des Néferkarê aux Montouhotep, 22, fig. 5). Voir aussi 78 no-0156 (infra, fig. 7).

35 R. Legros, "Approche méthodologique," 242 et fig. 8.

${ }^{36} C G C$, 21-22; PM, III.2, 565; Quibell, ExcavSaqq, 18-19, 25, pl. 9, 18. Cette table permet d'ailleurs de confirmer le lien entre la colonne d'inscription sur le pain et le cadre périphérique.

37 Il pourrait donc s'agir pour notre monument des périodes 6 à 8 de la sériation publiée au BIFAO, avec une probabilité peut-être plus forte pour la période 7.

${ }_{38}$ PN I, 298.17. Aucune des attestations données ne semble correspondre au même individu.

${ }^{39}$ Ce nom vient clairement de la racine $z \underline{t}$, "verser une libation” (WB III,422-23 et AnnLex 77.3375). Le terme est lié à un homonyme qui désigne le sol (voir aussi AnnLex 783316 et 79.2420). Il est significatif à cet égard que le déterminatif sur notre table ne représente pas l'eau, mais semble insister sur une action réalisée au niveau du sol. De fait, les tables d'offrandes retrouvées in situ ont quasiment toutes été déposées à même le sol (V. Dobrev et J. Leclant, "Les tables d'offrandes de particuliers découvertes aux complexes funéraires des reines près de la pyramide de Pépi I," dans N. Grimal, éd., Les critères de datation stylistiques à l'Ancien Empire: rencontre internationale, Institut français d'archéologie orientale, Le Caire, 10-13 novembre 1994, BdE 120 [Cairo, 1998], 151-52, fig. 2-3, 6-9; R. Legros, "La disparition d'une élite ? Les cultes privés dans la nécropole de Pépy I à Saqqâra,” dans J. Moreno García, éd., Élites et pouvoir en Égypte ancienne, CRIPEL 28 [Lille, 2009-2010], 157-59). Voir aussi la version profane "humidifier le sol."

${ }^{40}$ Dans le matériel découvert par la MafS, un autre bloc porte le même nom, pour un jmy-r wpt également, et appartient potentiellement au même individu (78 no-0497). Le déterminatif n'est pas lisible et les deux premiers signes sont inscrits dans l'ordre habituel. Un fragment de fausse-porte, pour un homonyme dont les titres ne sont pas conservés, porte également ce nom, inscrit cette fois selon la manière conventionnelle (Sc-385). 


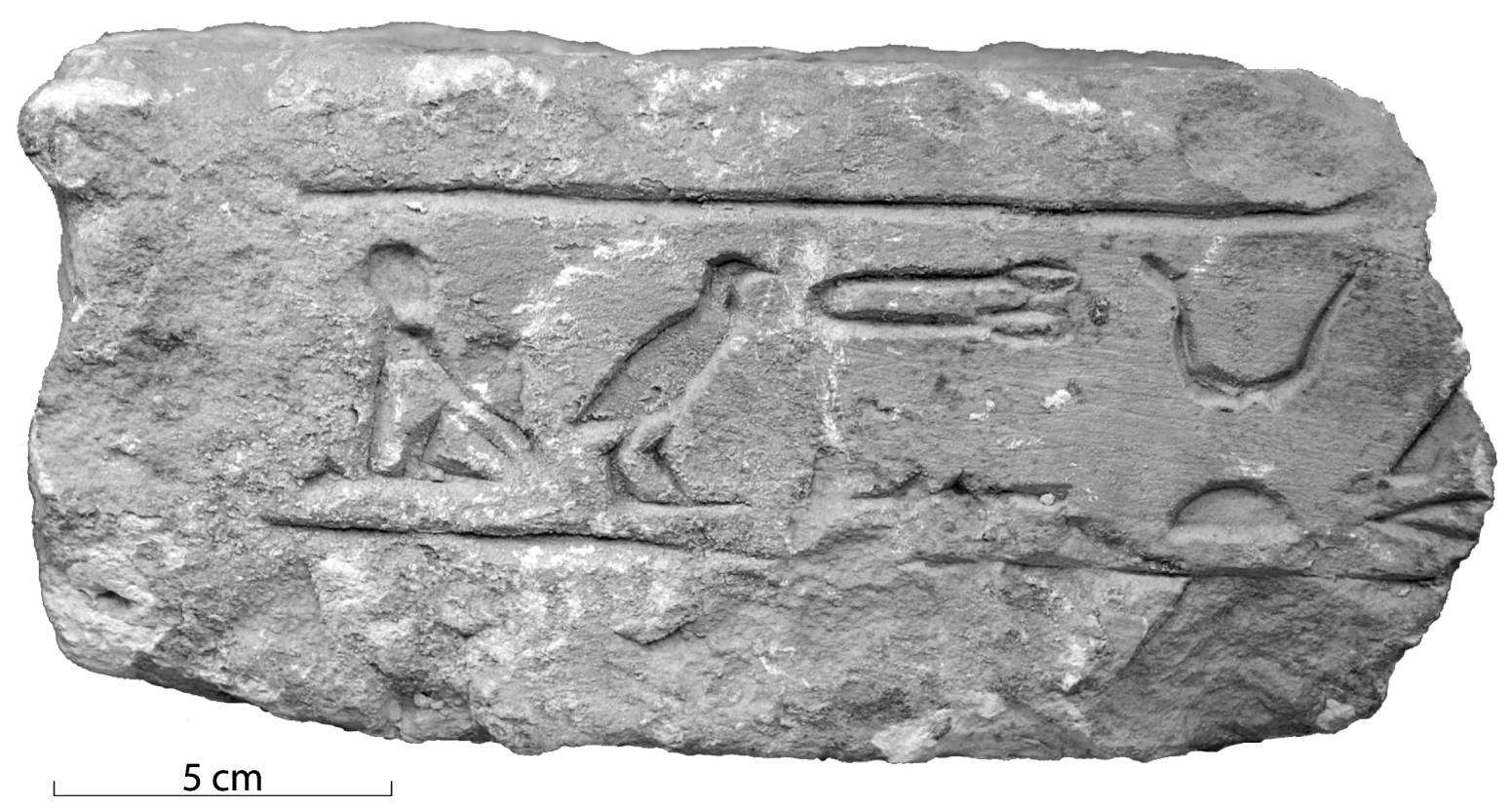

Fig. 2. Bloc inscrit au nom de l'imy-r wpt Sțw (inv. MafS 78 no-0092). @ M. Alavoine/Mafs.

Le principal titre porté par le défunt est celui de $h q 3$ h $h t$ "chef de district." ${ }^{11}$ Il devient particulièrement fréquent à partir du règne d'Isési et jusqu'à la fin de l'Ancien Empire. ${ }^{42} \mathrm{Il}$ s'agit d'un titre $a$ priori important, même si les conditions d'exercice de cette charge dans le courant de la PPI demeurent incertaines..$^{43}$ Deux $h q$ 3 $h$ $_{w t}$ du même nom sont déjà connus à Saqqâra. ${ }^{44}$

Le titre de hry-tp nswt "sous-ordre du roi" relève lui aussi d'une position sociale élevée. ${ }^{45}$ Associés à la mention smr $w^{\complement} t y$, ces deux titres constituent une séquence cohérente en terme de statut pour ce dignitaire.

Le propriétaire de cette table porte enfin deux titres d'imakhou, l'un en relation avec Anubis et l'autre avec Osiris. Pour ce dernier, la référence à $n \underline{t}$ ' $3 \mathrm{nb} p t$, comme s'il s'agissait d'une épithète, est sans parallèle à ma connaissance. Il pourrait s'agir de deux divinités distinctes, mais la juxtaposition serait tout aussi atypique. Dans notre documentation, les mentions du Grand Dieu avec l'épithète $n b$ $p t$ semblent se multiplier avec la fin de la PPI, ce qui rejoint l'analyse chronologique établie plus haut.

\section{Considérations sur la fabrication des tables d'offrandes}

Une observation singulière nous amène ici à évoquer les conditions de réalisation des tables d'offrandes : les deux moitiés de la table présentent des caractéristiques paléographiques propres, comme si le monument avait été réalisé par deux personnes différentes. Elles invitent à réfléchir d'une

${ }^{41}$ D. Jones, An Index of Ancient Egyptian Titles, Epithets and Phrases of the Old Kingdom, BAR Int. Series 866 (Oxford, 2000), 670 [2453]; pour une liste des attestations, voir Moreno García, Hwt et le milieu rural, 270-77.

${ }^{42}$ Moreno García, Hwt et le milieu rural, 229-32, 241. Pour la distribution géographique des attestations de ce titre et le poids de la région memphite, cf. ibidem, 252-53.

${ }^{43}$ Les attestations deviennent très rares à partir de la $11^{\mathrm{e}}$ dynastie (Moreno García, Hwt et le milieu rural, 266, 269) et notre table est sans doute antérieure de très peu à cette période (cf. infra).

${ }^{44}$ Moreno García, Hwt et le milieu rural, 272.

${ }^{45}$ Jones, Index, 788 [2874]. 
manière plus générale sur le processus de réalisation des monuments de culte privé et sur l'éventualité d'un travail en équipe.

Ces variations que l'on peut observer entre les deux moitiés du monument relèvent certes du détail, mais nous semblent trop systématiques pour ne pas être évoquées. Un tableau comparatif permet de mettre en évidence les différences les plus notables.

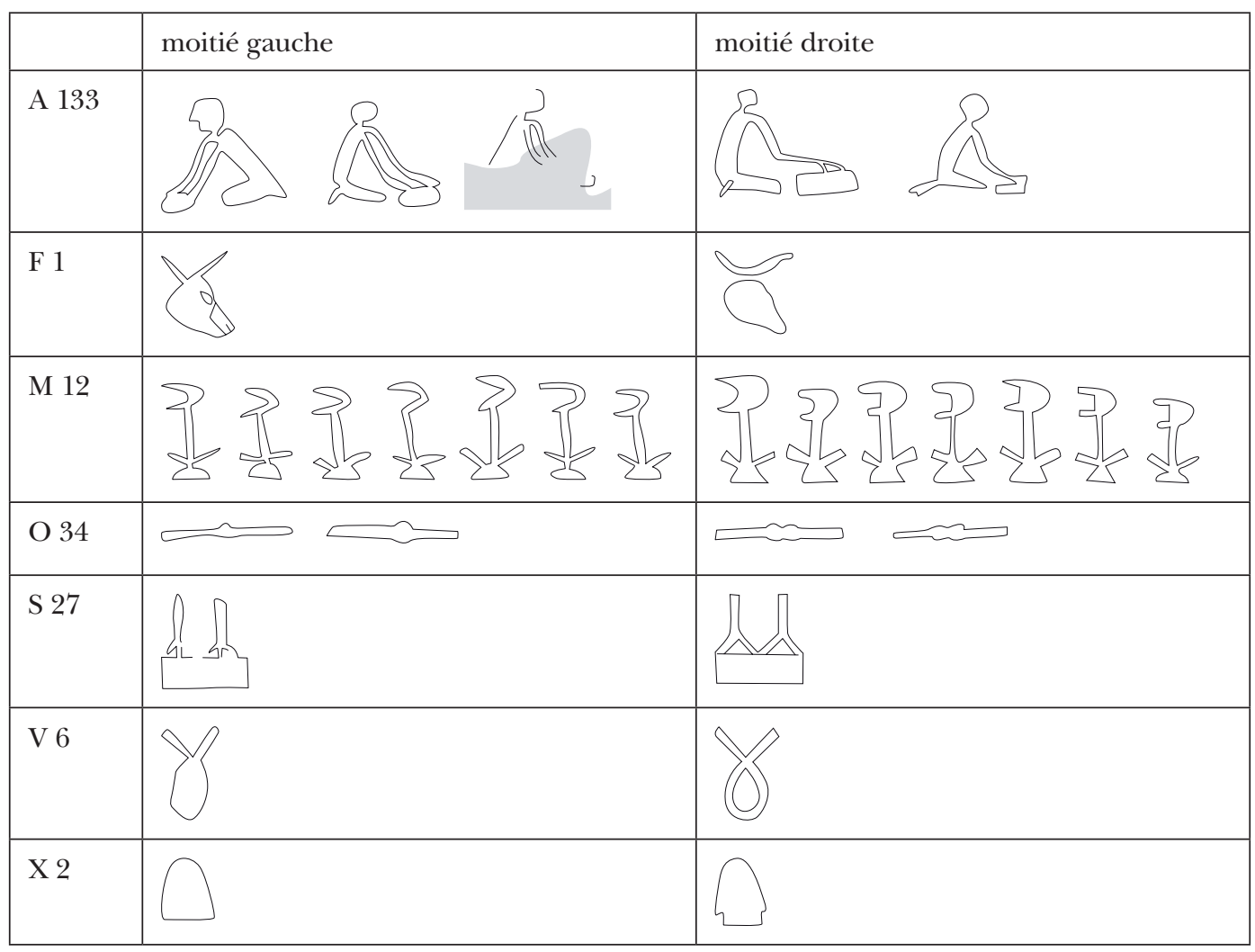

L'hypothèse d'une réalisation double est venue d'abord de la graphie du nom du propriétaire, qui présente systématiquement les mêmes variantes : à droite, le déterminatif montre toujours les deux bras alors qu'il n'y en a qu'un seul à gauche; de même, le signe $s$ est toujours écrit avec les deux ronds centraux à droite, mais un seul à gauche. De ce point de vue, la graphie sur le pain se rapproche donc de celle de la partie droite.

Dans la liste d'offrandes, le signe des milliers (M12) présente une variation au niveau de la fleur, qui peut former un véritable arc de cercle (à gauche) ou un volume plus plein (à droite). Pour la tête de bœuf (F1), au delà des détails intérieurs, moins significatifs, c'est plutôt la représentation des cornes qui présente une variation intéressante, tant pour la courbure que pour l'accroche au sommet du crâne. Le signe de l'albâtre apparaît comme un sac à gauche, entièrement évidé, alors qu'il s'agit bien d'un lien sur la moitié droite. Le signe S27 enfin est mal conservé sur la moitié gauche. Il semble bien toutefois que les deux montants verticaux se terminent par une fourche tripartite, ce qui par ailleurs est un cas unique à notre connaissance.

Dans la formule prt-hrw, la forme du pain $t(\mathrm{X} 2)$ est aussi différente des deux côtés.

Enfin, bien que moins évident, on pourrait également évoquer la tête de canard, dont la forme est identique des deux côtés, mais qui mesure seulement un demi quadrat de hauteur à droite alors qu'elle occupe un quadrat complet à gauche. L'interversion des signes $b$ et st dans bnrt ne se retrouve peut-être pas sur la moitié gauche, mais une lacune du signe empêche d'être définitif sur ce point. 
Les différences entre les deux moitiés sont donc nombreuses et significatives. Il n'est pas exclu qu'il s'agisse d'un artisan peu constant, mais le caractère répété de certaines variantes va plutôt dans le sens d'une réalisation collective, avec deux mains bien distinctes.

Si l'on dépasse maintenant le cadre de l'étude propre à cette table, il est possible d'émettre un certain nombre d'hypothèses complémentaires sur les modalités de réalisation de ces objets de culte privé et sur l'existence d'ateliers de fabrication.

\section{La provenance du matériau}

La question du matériau tout d'abord n'est pas sans intérêt. La plupart des tables d'offrandes et des fausses-portes retrouvées par la MafS sont réalisées dans un calcaire fin qui ne correspond pas à celui que l'on trouve naturellement sur le site. ${ }^{46}$ En effet, la fouille des pyramides de la nécropole de Pépy I ${ }^{\text {er }}$ permet de distinguer clairement les blocs de calcaire local utilisés pour le blocage interne, de qualité médiocre, et ceux employés pour les parements, bien plus fins, et importés de carrières extérieures. Les monuments privés relèvent quasiment tous de cette deuxième catégorie.

Aucun espace spécifique n'a été retrouvé à ce jour qui pourrait être interprété comme un atelier de fabrication. Il est certain cependant qu'il devait s'en trouver un car plusieurs blocs retrouvés hors contexte témoignent d'un travail en cours de réalisation (fig. 3). ${ }^{47}$

Il n'est donc pas exclu que les petits monuments ait été réalisée sur le site même. On peut même raisonnablement émettre l'hypothèse qu'une partie au moins de ces objets aient été réalisés à partir des rebuts de construction qui devaient se trouver à proximité en quantités importantes, en raison de la difficulté pour les bâtisseurs à estimer la quantité de matériaux nécessaires, des retouches/retailles pour ajuster les blocs, etc... La permanence du chantier de construction pendant les règnes de Pépy I et II, voire au delà, constituerait un cadre particulièrement propice au développement de cette activité. En outre, le poids des tables les plus grandes devait inciter à réduire les déplacements à leur minimum. ${ }^{48}$

\section{La préparation}

Chaque bloc devait être préparé de manière à recevoir l'inscription prévue: dégrossissage, mise en place des éléments de reliefs (pain, bassins, bandeau périphérique, apex...) puis polissage de la surface. On observe sur cette dernière étape des différences de qualité assez variées allant du bloc presque brut, y compris sur la face inscrite, jusqu'aux tables très soignées, même sur les bords et en dessous. Il faut donc envisager deux hypothèses: soit le destinataire final était connu dès cette étape et l'objet était travaillé en fonction de la demande ou du "prix" annoncé; soit les objets étaient réalisés en série, avec différents degrés de qualités pour pouvoir répondre aux multiples besoins.

De nombreuses tables d'offrandes ont été retrouvées sans aucune inscription dans la nécropole de Pépy $\mathrm{I}^{\text {er }}$. Il est difficile de dire si elles étaient ou non en cours de réalisation, destinées à être inscrites par la suite, ou bien s'il s'agit de monuments qui devaient rester anépigraphes. Cette dernière hypothèse ne peut être complètement écartée car, sur la centaine de tables retrouvée in situ, trois étaient effectivement anépigraphes.

Parmi les étapes de préparation, il faut également évoquer la mise en place du texte. Le fait est bien connu, pour les tombeaux de particuliers comme pour les textes des pyramides, qu'un tracé préliminaire était réalisé pour mettre en place les éléments de la composition. Pour les tables d'offrandes, rien

\footnotetext{
${ }^{46}$ Cependant, lors d'une visite de sa fouille, K. Mysliwiec faisait remarquer que la qualité des pierres dans le $d r y-m o a t$ varie selon les couches.

47 Il n'est pas exclu que certains de ces objets, dont la forme très ordinaire se retrouve à toutes les époques, datent en fait du pillage des pierres de la nécropole à partir du Nouvel Empire. Une telle pratique se retrouve d'ailleurs sur la plupart des complexes royaux de Saqqâra: on voyait encore il y a peu une ébauche de statue cube au temple d'Isési.

${ }^{48}$ Pour donner un ordre de grandeur, une table comme celle de Sétjou avoisine les $100 \mathrm{~kg}$.
} 

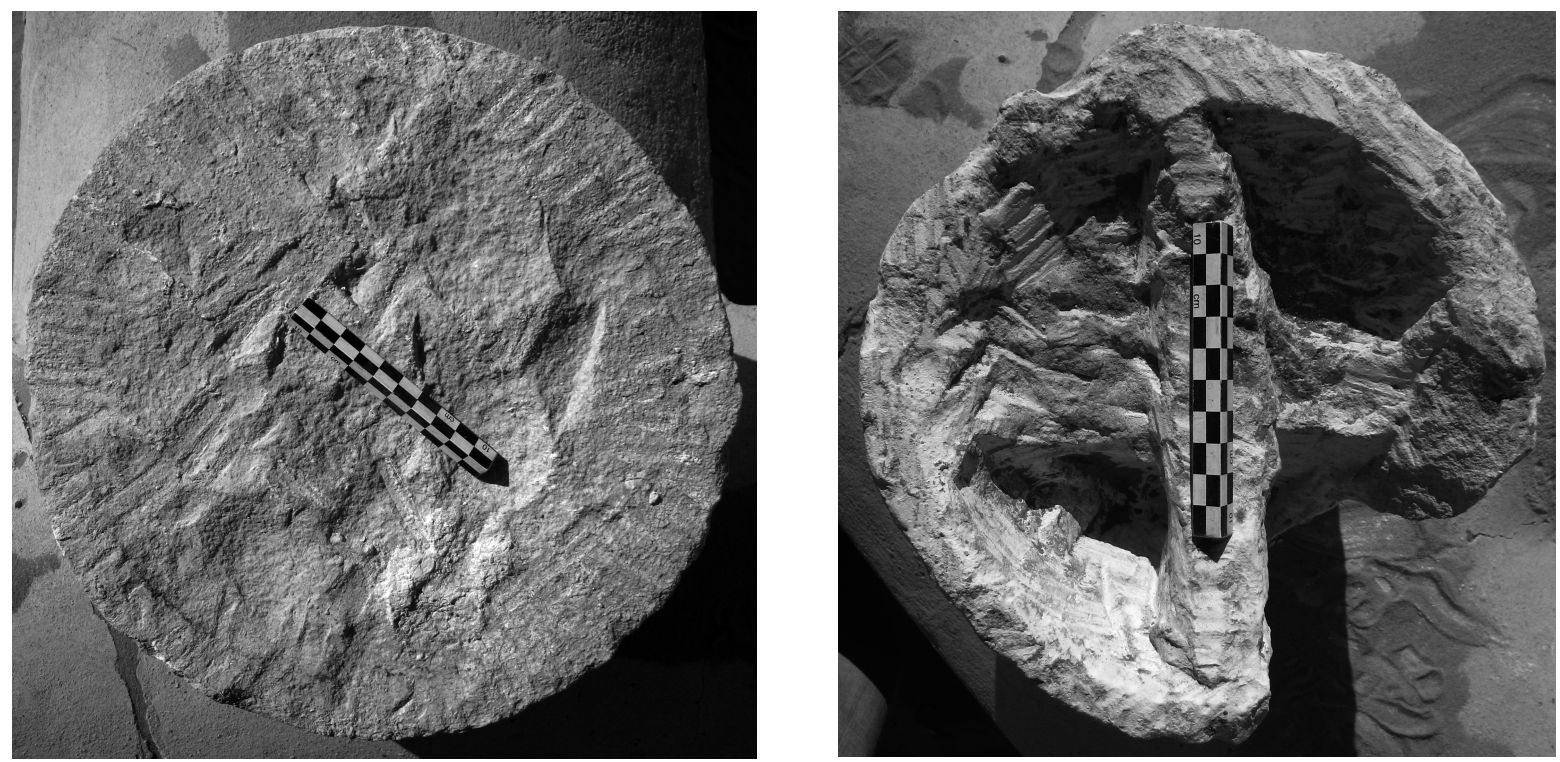

Fig. 3a-b. Deux vasques en cours de réalisation, à des stades différents $\left(\right.$ sans $\mathrm{n}^{\circ}$ ). Celle de gauche conserve la marque au trait noir de l'espace à évider.
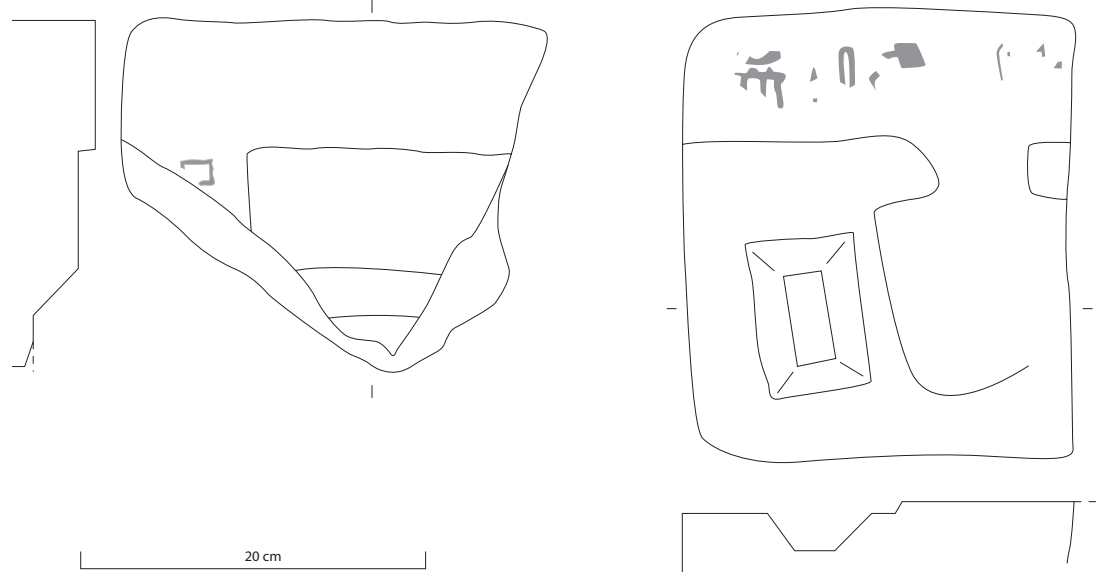

Fig. 4. Fragments de tables d'offrandes avec signes peints (inv. MafS 09-194 et 78 no-0989).

d'explicite n’a été retrouvé à ce jour, toutes les pièces retrouvées étant uniformément vierges de couleur. Deux blocs toutefois, bien modestes, présentent les restes d'un texte peint (fig. 4). L'inscription est illisible dans les deux cas, mais assure néanmoins l'existence de peinture également sur ce type de monuments. La question demeure en revanche entière de savoir s'il s'agit de tracés préparatoires ou d'inscriptions supposées être définitives. ${ }^{49}$

${ }^{49}$ Le tracé préparatoire sur table d'offrandes est attesté pour d'autres périodes, notamment la Basse Époque (cf. G. Martin, The Tomb of Hetepka and other Reliefs and Inscriptions from the Sacred Animal Necropolis, North Saqqara, 1964-1973, EESTEM 4 [London, 1979], 71, $\mathrm{n}^{\circ}$ 266). Une seule table à ma connaissance (pour un contexte proche) porte des traces de peinture, en l'occurrence un fond jaune: Firth-Gunn, Teti Pyramid Cemeteries, 221, n²22. Voir aussi M. Bárta, "An Old Kingdom book for the 


\section{L'inscription}

La gravure de l'inscription fait sans doute l'objet d'une répartition des tâches. Comme il a été dit ci-dessus, la réalisation de la table d'offrandes de Sétjou a vraisemblablement être divisée selon deux moitiés, inscrites par des personnes différentes. Il s'agit d'un cas unique à notre connaissance de travail en binôme sur deux moitiés d'une même table, mais d'autres monuments témoignent de réalisations multiples ou en plusieurs temps.

On connait bien le principe du rajout d'inscription, lorsqu'un individu vient compléter un monument préexistant en y apposant son propre nom. Le phénomène est attesté sur toute sorte de supports et quelques exemples peuvent aussi se retrouver sur les tables d'offrandes (fig. 5) ${ }^{50} \mathrm{~S}$ 'il s'agit bien ici de deux mains différentes - et c'est d'ailleurs à cela que l'on reconnait l'ajout - le principe est néanmoins un peu différent dans la mesure où la conception originelle du monument ne prévoyait pas cette modification. Cette caractéristique ne peut donc être prise en considération dans la discussion sur le travail collectif.

Plus intéressant pour notre propos, il existe des objets dont l'inscription est mal disposée, c'est à dire "débordant" de l'espace qui lui est normalement dévolu. Dans le cas des tables d'offrandes, cette erreur d'anticipation de la longueur de l'inscription est particulièrement flagrante en bout de natte (fig. 6). ${ }^{51}$ En cet endroit, l'inscription porte traditionnellement de droite à gauche une formule d'offrandes suivie des titres du défunt puis de son nom. Il n'est pas rare que ce dernier, faute de place, soit relégué entre la natte et le bassin.

L'erreur d'appréciation dans la gestion de l'espace et l'organisation des cadrats peut être due à l'absence de tracé préparatoire. On peut également envisager une inscription en plusieurs étapes avec, dans un premier temps, la gravure des formules d'offrandes stéréotypées, de manière à préparer le monument avant même qu'il soit attribué à une personne en particulier. La fin de l'inscription, en fonction des titres et de la longueur du nom du bénéficiaire, ne peut alors correspondre systématiquement à la place qui leur a été réservée.

Cette hypothèse semble confortée par certaines inscriptions dont la partie gauche apparaît tassée, pour éviter précisément ce type de débordement, alors que la partie droite est correctement disposée (fig. 7).$^{52}$ Le plus significatif dans cette disposition est que la rupture entre la partie droite - aux cadrats larges et aérés - et la partie gauche, dont les signes sont entassés, se trouve immédiatement et systématiquement après la formule d'offrandes prt-hrrw. Une situation similaire a déjà été reconnue pour une fausse porte de Giza, là aussi avec une rupture entre les formules rituelles et les titres..$^{53} \mathrm{Il}$ nous semble qu'il y a là un indice fort en faveur d'une préparation des inscriptions avant leur affectation à un destinataire spécifique. L'auteur des deux parties n'est pas nécessairement différent et il peut tout à fait s'agir du même artisan qui réalise le monument en deux temps successifs. Pour autant, l'hypothèse d'un travail à plusieurs, au sein d'un atelier ne peut être complètement rejetée, notamment si l'on admet que la table de Sétjou a bien été réalisée par deux mains distinctes.

afterlife," dans D. Magee, J. Bourriau, S. Quirke, eds., Sitting beside Lepsius. Studies in Honour of Jaromir Malek at the Griffith Institute (Louvain, Paris, 2009), 30-31.

${ }^{50}$ Cette table a été retrouvée in situ (cf. R. Legros, "disparition d’une élite," fig. 3). Elle date sans doute du règne de Pépy $\mathrm{I}^{\mathrm{er}}$ : figure debout avec bâton sur la natte, double ligne, chacal sur signe $h t p$, formule prt-hrww avec pronom suffixe, mention de la tombe et d'une fête. Le signe de la nécropole (R10) avec une projection à la base du mât, se développe à partir de la $6^{\mathrm{e}}$ dynastie (H. Fischer, "The Evolution of Composite Hieroglyphs in Ancient Egypt," MMJ 12 (1978), 8-9 ; idem, Varia Nova, Egyptian Studies 3, [New York, 1996], 27).

${ }^{51}$ Cette table date sans doute des périodes 5 à 7 en raison de la petite table à pains devant le défunt. Le signe $m r$ sans le lien médian est rare (voir la stèle Univ. Coll. 14321: Fischer, Coptite Nome, 101, fig. 14, pl. 35b). Pour le signe pr sans les murs de retour, voir D. Meeks, Les architraves du temple d'Esna. Paléographie, PalHiéro 1 (2004), 147, § 395.

${ }^{52}$ Cette table ne peut être datée que largement, des périodes 3 à 7: table "flottante" devant le défunt, forme du fauteuil.

53 Der Manuelian, "Case of Prefabrication," 115-27. 

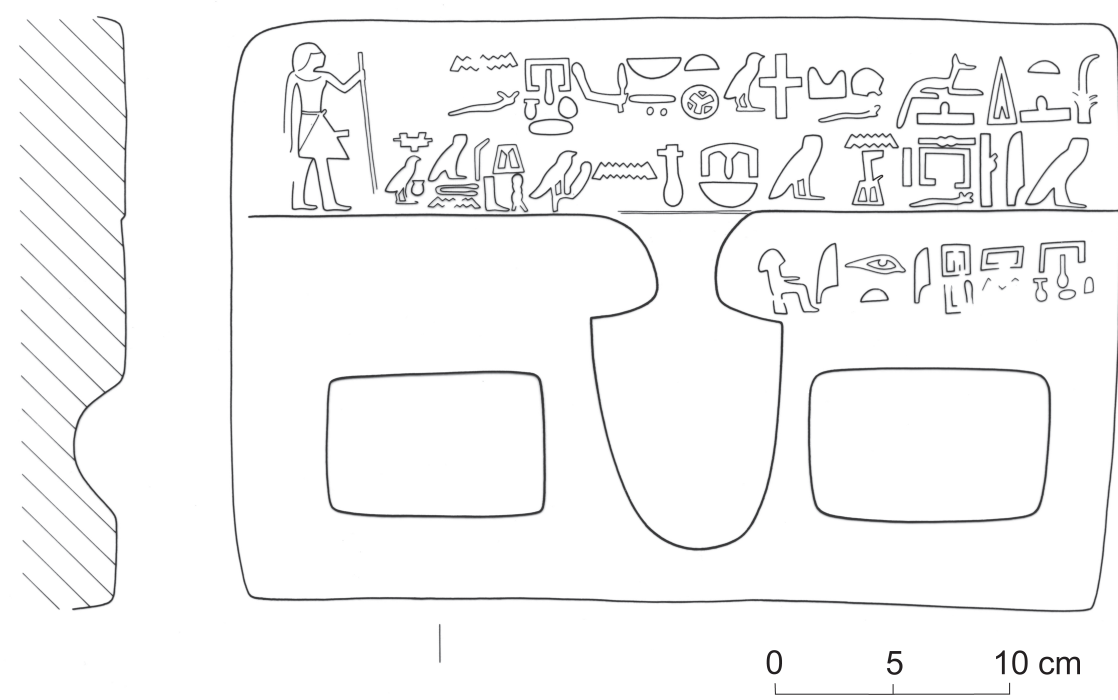

Fig. 5. Table Sc-192, avec rajout d'une inscription postérieure entre la natte et le bassin (les signes pr et X, notamment, semblent indiquer une autre main). (C) I. Pierre-Croisieau/R. Legros.

\section{Approche quantitative}

Pour conclure sur le contexte de réalisation des tables d'offrandes et l'existence d'ateliers, une approche quantitative permet de placer cette documentation sous un jour particulier. Un inventaire des tables d'offrandes a été réalisé, aussi systématique que possible, pour la période allant du début de la $6^{\mathrm{e}}$ dynastie à la fin de la $10^{\mathrm{e}}$ dynastie, incluant les découvertes de la MafS sur la nécropole de Pépy I ${ }^{\mathrm{er}}$, mais aussi les tables trouvées en différents secteurs de la nécropole de Saqqâra.

La nécropole de Pépy I ${ }^{\text {er }}$ a livré à ce jour 832 fragments (après assemblage) pour un nombre minimum d'au moins 543 monuments. ${ }^{54}$ Le nombre réel de monuments est donc à situer entre les deux chiffres. Dans la pratique, de nombreux assemblages ont été réalisés ou tentés de manière systématique et l'éventualité de nouveaux raccords est assez peu probable. Les autres secteurs de la nécropole de Saqqâra ${ }^{55}$ ont livré conjointement 235 monuments. Pour ceux là, il n'est pas possible de savoir si certains fragments modestes ont été négligés ou non (G. Jéquier le laisse entendre à plusieurs reprises pour la nécropole de Pépy II).

La durée de la période considérée, de l'avènement de Téti ( $c a$ 2340-2300) à la réunification sous Montouhotep II ( $c a$ 2000-1960), représente potentiellement un peu plus de trois siècles. ${ }^{56}$ Cette donnée permet de relativiser l'importance d'un éventuel atelier: s'il s'agit de fait d'un chiffre considérable dans l'absolu, approchant le millier d'individus, une fois rapporté au nombre d'année, cela ne représente plus que deux ou trois monuments par an en moyenne. Il faut donc supposer - ce qui est d'ailleurs assez logique - que les artisans travaillaient sur plusieurs types de monuments et que la réalisation des tables d'offrandes n'était pas un travail spécifique et détaché du reste de l'activité de gravure. Si atelier il y eut, il devait probablement produire toute sorte d'autres monuments.

${ }^{54}$ Ce chiffre est basé sur le décompte des angles conservés.

${ }^{55}$ Principalement la nécropole de Pépy II et celle de Téti.

${ }^{56}$ L'étendue chronologique de la PPI est encore très controversée. On retient ici la proposition de J. von Beckerath, Chronologie des Pharaonischen Ägypten, MÄS 46 (Munich, 1997), 188: il ne s'agit pas de chercher une datation précise, mais un ordre de grandeur. 


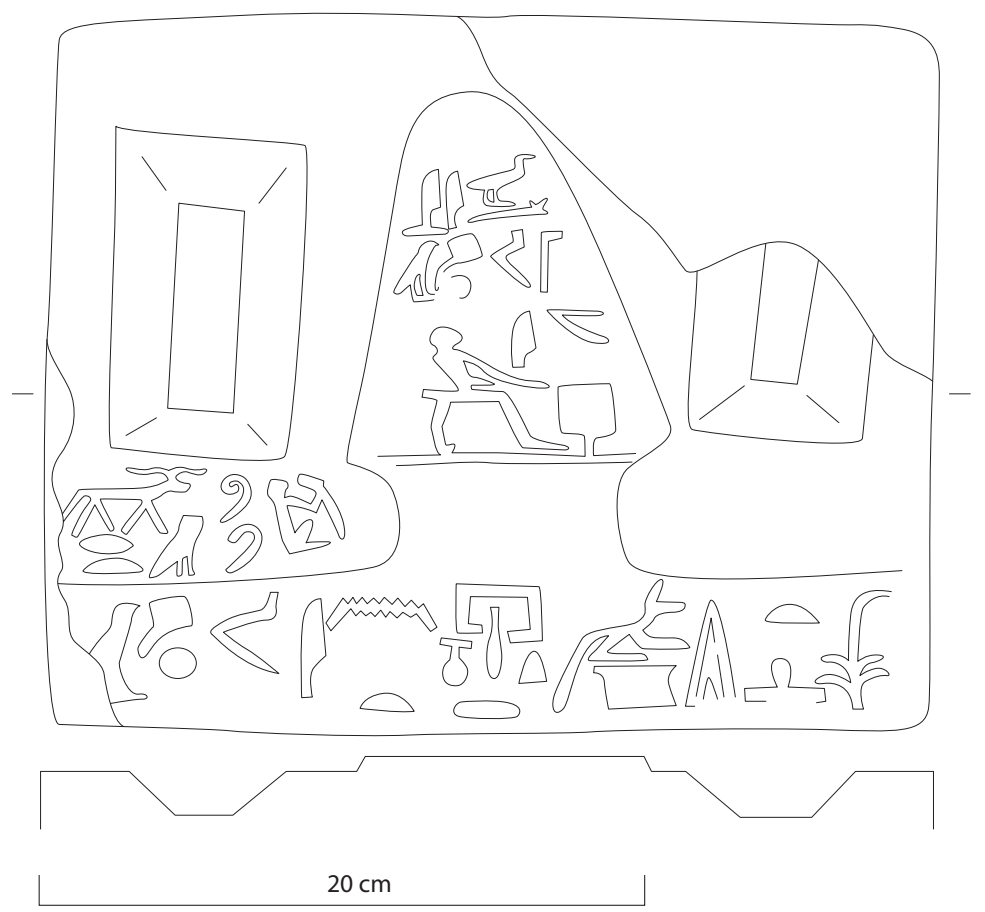

Fig. 6. Table Sw-256, avec mauvaise gestion de la longueur de l'inscription.

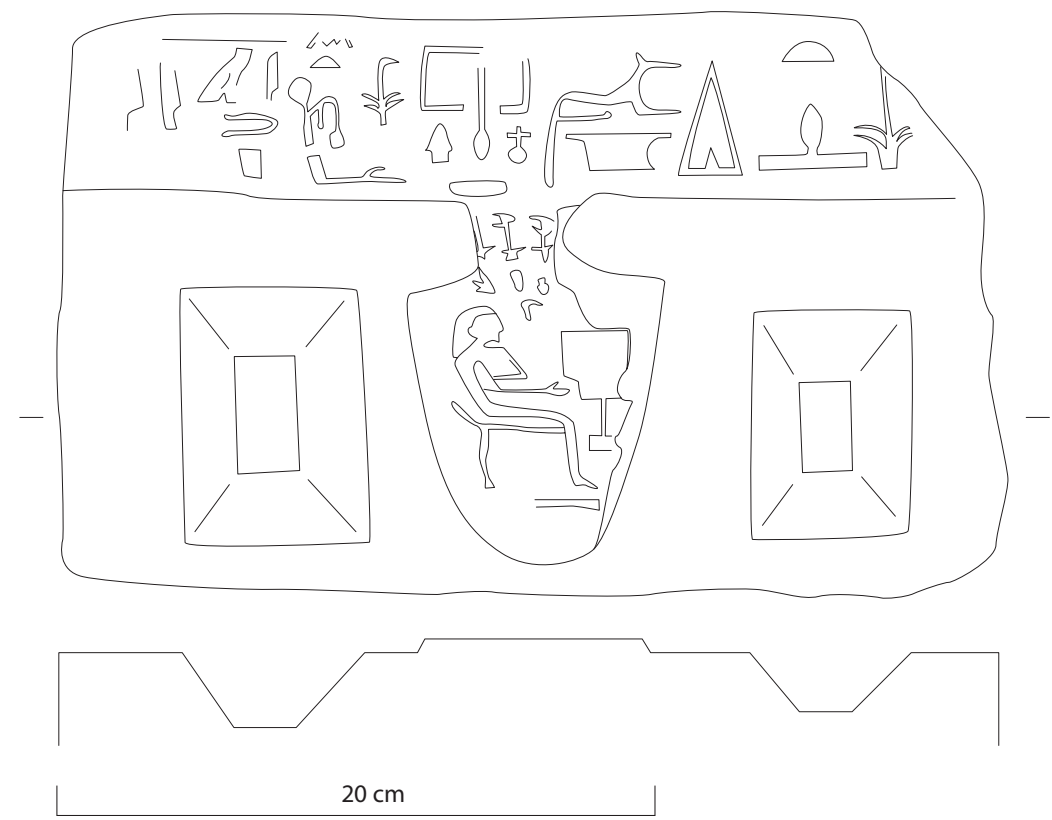

Fig. 7. Table 78 no-0156 du š́ ${ }^{c}$ nswt Impy, avec "compression" des cadrats en fin de natte. (C) M.-N. Fraisse/R. Legros. 
L'écart important entre le nombre d'objets découverts dans la nécropole de Pépy I ${ }^{\text {er }}$ et les autres secteurs du site de Saqqâra appelle également un commentaire, d'autant plus si l'on tient compte du fait que les premiers règnes de la $6^{\mathrm{e}}$ dynastie ne sont pas attestés sur la nécropole de Pépy ${ }^{\mathrm{er}}$. La présence d'un atelier de fabrication à Saqqâra sud ne peut être garantie en l'absence de vestige explicite, mais plusieurs indices vont dans ce sens. La découverte de quelques blocs en cours de fabrication (voir supra) constitue bien sûr un élément important. Quant au nombre des tables, il nous renseigne avant tout sur le lieu du dépôt, mais ne peut garantir celui de leur réalisation. Il faut pourtant considérer que l'activité sur cette zone, sans équivalent pour le nombre des pyramides construites et la continuité des rites, fournit le cadre idéal pour l'implantation d'un atelier qui, installé à proximité, bénéficierait à la fois des matériaux nécessaires, en provenance des chantiers de construction, de la présence de nombreux dévots et des conditions rituelles propices à l'utilisation des objets réalisés.

Au sein d'un tel atelier, le travail des graveurs fait l'objet apparemment d'une répartition des tâches. Celle-ci peut être d'ordre chronologique avec différentes étapes successives dans la préparation du support et l'inscription des textes. Cette répartition peut aussi relever d'une organisation des équipes, avec plusieurs individus qui travaillent simultanément ou en alternance sur les mêmes monuments.

Mission archéologique française de Saqqâra Laboratoire HiSoMA-CNRS 